\title{
LINFOMA MULTICÊNTRICO CANINO: uma sinopse sobre os aspectos clinicopatológicos e alterações laboratoriais
}

\author{
Canine multicentric lymphoma: a synopsis of clinicopatholo- \\ gical aspects and laboratory changes
}

\author{
Mariana Ramos Andrade Beraldo ${ }^{1 *}$; Fernanda L. S. B. Varzim²; Lidia Hildebrand Pulz ${ }^{3}$ \\ *Autor Correspondente: Mariana Ramos Andrade Beraldo, Rua Gabriel Ferreira, 213, Centro, \\ Águas da Prata, SP, Brasil - CEP.: 13890-000. E-mail: mraberaldo@gmail.com
}

\begin{abstract}
T Como citar: BERALDO, Mariana Ramos Andrade; VARZIM, Fernanda L. S. B; PULZ, Lídia Hildebrand. Linfoma multicêntrico canino: uma sinopse sobre os aspectos clinicopatológicos e alterações laboratoriais. Revista de Educação Continuada em Medicina Veterinária e Zootecnia do CRMV-SP, São Paulo, v.18, n. 2, 2020. Doi 10.36440/recmvz.v18i2.38066
\end{abstract}

Cite as: BERALDO, Mariana Ramos Andrade; VARZIM, Fernanda L. S. B; PULZ, Lidia Hildebrand. Canine multicentric lymphoma: a synopsis of clinicopathological aspects and laboratory changes. Journal of Continuing Education in Animal Science of CRMV-SP, São Paulo, v.18, n.2, 2020. Doi 10.36440/recmvz.v18i2.38066

\section{Resumo}

O linfoma é uma neoplasia caracterizada pela proliferação de células linfoides malignas, que pode se desenvolver em qualquer órgão, acometendo inicialmente linfonodos, baço e fígado. Sua causa não é bem elucidada, porém acredita-se na etiologia multifatorial, fatores genéticos, deficiência imunológica e carcinógenos químicos. Existem quatro classificações anatômicas para o linfoma: multicêntrico, alimentar ou digestiva, mediastínico e extranodal, sendo que $80 \%$ dos casos em cães são classificados como linfomas multicêntricos. 0 diagnóstico é baseado em análises citológicas, histológicas e biologia molecular, que auxiliam no estabelecimento do prognóstico do animal considerando o grau de malignidade do linfoma e o tipo celular de origem. Os exames laboratoriais, como os perfis hematológicos e bioquímicos, fornecem dados para o acompanhamento do animal, acometimento e estadiamento da doença. Perante o exposto, constata-se que o linfoma é uma afecção de grande relevância dentre aquelas que acometem cães, portanto, este estudo tem o objetivo de aliar a base teórica frente à apresentação clínica do linfoma, fornecendo o conhecimento das principais alterações descritas para gerar um diagnóstico precoce, estabelecendo uma ponte de sucesso com o clínico e permitindo ao paciente melhor qualidade de vida e sobrevida perante a doença.

Palavras-chave: Cães. Linfoma. Neoplasia.

1 Aprimoranda em Propedêutica Complementar, Hospital Veterinário Vicente Borelli, Centro Universitário Fundação de Ensino Octávio Bastos (Unifeob), São João da Boa Vista, SP, Brasil

2 Docente e orientadora da disciplina Propedêutica Complementar, Centro Universitário Fundação de Ensino Octávio Bastos (Unifeob), São João da Boa Vista, SP, Brasil

3 Docente e orientadora da disciplina Propedêutica Complementar, Centro Universitário Fundação de Ensino Octávio Bastos (Unifeob), São João da Boa Vista, SP, Brasil 


\section{Abstract}

Lymphoma is a tumor characterized by the proliferation of malignant lymphoid cells, which can occur in any organ, initially affecting lymph nodes, spleen and liver. It's cause is not elucidate, but it might be a multifactorial etiology, including genetic factors, immune deficiency and chemical carcinogens. There are four anatomical classifications for lymphoma: multicentric, alimentary, mediastinal and extranodal, but, $80 \%$ of the cases in dogs are classified as multicentric lymphomas. The diagnosis is based on cytological, histological and molecular biology sample analyzes, that assist in settlement the animal's prognosis considering the degree of malignancy of the lymphoma and the cell of origin. Laboratory tests such as hematological and biochemical profiles provide data for monitoring the animal, disease involvement and staging. Lymphoma is a condition of great relevance within the conditions that affect dogs, making it necessary to educate veterinarians to combine the academic basis with the clinical presentation of lymphoma, with the knowledge of the main changes described to create an early diagnosis, establishing a conjunction of success with the clinical and allowing the patient a better quality of life and survival in the face of the disease.

Keywords: Dogs. Lymphoma. Neoplasia.

\section{Introdução}

O linfoma, distúrbio linfoproliferativo frequente nos pequenos animais, é uma neoplasia de alta incidência entre todos os tipos de neoplasias malignas nos cães, representa cerca de $24 \%$ do total das neoplasias caninas e 83\% das neoplasias hematopoiéticas (VAIL; OGILVIE, 2003).

0 linfoma é definido como uma proliferação clonal de linfócitos malignos nos órgãos linfoides como medula óssea, baço e linfonodos (VIEIRA, 2013). Em cães apresenta diferentes subtipos e tipos histológicos originários das fases da ontogenia dos linfócitos B e T (KIMURA, 2012).

A causa do linfoma não está totalmente elucidada, porém acredita-se na etiologia multifatorial que envolve fatores genéticos, deficiência imunológica, e carcinógenos químicos. Os sinais clínicos apresentados pelo cão com linfoma são inespecíficos e dependem do local anatômico afetado e da extensão da doença (FERREIRA; AZEVEDO; LEITE, 2015).

O diagnóstico definitivo do linfoma multicêntrico é obtido com a análise histopatológica e citológica dos órgãos e linfonodos acometidos, entretanto, para melhor compreensão do comportamento biológico tumoral e precisão diagnóstica, sugere-se a utilização das técnicas de biologia molecular ou imuno-histoquímica. O diagnóstico presuntivo ou suspeita pode ser estabelecido frente ao exame físico, histórico do animal, alterações laboratoriais e de imagens (FERREIRA; AZEVEDO; LEITE, 2015; DICKINSON, 2008) Diante do exposto o presente trabalho faz uma revisão sobre o linfoma multicêntrico canino, dando ênfase nos aspectos clínico-patológicos e nas principais alterações laboratoriais, descritas com o propósito de colaborar com o entendimento do clínico veterinário frente ao diagnóstico desta doença.

\section{Discussão}

O linfoma é uma neoplasia originária de tecidos linfoides, caracterizado pela proliferação de células linfoides malignas, podendo se desenvolver em qualquer órgão, o que difere das leucemias que tem origem na medula óssea (VALERIANO et al., 2017; FERREIRA; AZEVEDO; LEITE, 2015). 0 linfoma é considerado a neoplasia mais comumente tratada em cães, o que contribui por ser uma doença de alta incidência na população mundial canina (CÁPUA et al., 2011). Considerando o acometimento apenas em cães idosos, a sua prevalência pode chegar a 84 por 100.000 cães, e naqueles com menos de um ano de idade, a prevalência cai para 1,5 por 100.000 cães (VAIL; MACEWEN; YOUNG, 2001). 
A causa do linfoma ainda não está bem estabelecida, mas alguns fatores podem contribuir para o desenvolvimento da doença, como o aumento da sua ocorrência em cães com doenças autoimunes, por exemplo, a trombocitopenia imunomediada, que afeta cães independente do sexo e idade, têm sido associada a um maior risco de desenvolvimento do linfoma quando comparada com a população saudável. Contudo, uma relação de causa e efeito ainda não foi comprovada, porém pode estar relacionada predisposições raciais, animais consanguíneos e produtos químicos (SILVA; SEQUEIRA, 2016; VALERIANO et al., 2016; OLIVEIRA, 2014). Quando comparados aos humanos aqueles que possuem imunossupressão são os que apresentam maior risco de desenvolver neoplasias no sistema linforreticular, acredita-se que, nesses pacientes, possa ter ocorrido uma super expressão de alguns oncogenes em linfócitos, que poderiam propiciar o desenvolvimento da neoplasia (TESKE, 1994).

A classificação do linfoma multicêntrico é geralmente baseada em critérios anatômicos, histológicos, características citológicas e imunofenotípicas. Os linfomas caninos apresentam características semelhantes às dos linfomas não Hodgkin (GREENLEE et al., 1990; TESKE, 1994). Os linfomas podem também ser divididos de acordo com sua origem em linfomas de células $B$ e de células T, sendo que o pior prognóstico é apontado para os de células T (SILVA; SEQUEIRA, 2016).

Atualmente, a imunofenotipagem passou a ser indispensável para a determinação o diagnóstico e o prognóstico de linfoma não Hodgkin. Nos cães, são utilizados os mesmos critérios de classificações aplicados aos linfomas não Hodgkin humanos e as classificações atualmente adotadas são as de: Kiel (LENNERT; FELLER, 1992), REAL (HARRIS et al., 1994) e WHO (JAFFE et al., 2001).

o Sistema Kiel, baseia-se na morfologia e composição celular (centroblástica, centrocítica, imunoblástica, linfoblástica, plasmocítica, linfocítica), no padrão de crescimento histológico (folicular ou difuso) e na imunofenotipagem das células tumorais, B e T. Nesta classificação, o linfoma é dividido em dois grupos: a) de baixo grau, constituídos principalmente por células pequenas (citos) e b) de alto grau, constituídos predominantemente por células médias ou grandes (blastos) (LENNERT; FELLER, 1992). Este sistema divide também o linfoma em dois subgrupos, tendo por base a imunofenotipagem (B e T) (VALLI et al., 2002).

Em 1994, o International Lymphoma Study Group, a Revised European-American Classification of Lymphoid Neoplasms (REAL) (HARRIS et al., 1994) propôs uma nova classificação para o linfoma. Esta proposta, mais abrangente, leva em conta as características imunológicas, as alterações genéticas e as características clínicas dos tumores. Esta abordagem foi posteriormente adotada, com pequenas modificações, pela Organização Mundial de Saúde - World Health Organization (WHO) e introduzida como WHO Classification of Tumors of the Hematopoietic and Lymphoid Tissues em 2001 e 2008 (GOOD; GASCOYNE, 2008). A classificação REAL-WHO mantém a morfologia como critério básico (VALLI, 2007). Em 2002, foi publicada a Classificação Histológica de Tumores Hematopoiéticos de Animais Domésticos, que se baseou na classificação da WHO permitindo a maior facilidade na comunicação entre os patologistas humanos e veterinários, tanto para propósitos de diagnóstico como de pesquisa (VALLI et al., 2002). Nestes sistemas os tumores são classificados em alto ou baixo grau de malignidade, sendo que os linfomas com baixo grau são os constituídos por células pequenas com baixo índice mitótico, que geralmente progridem lentamente e são menos responsivos a quimioterapia, quando comparados ao linfoma de alto grau, porém estão associados a maior tempo de sobrevida. Os linfomas classificados de alto grau de malignidade têm um elevado índice mitótico, este tipo de linfoma progride rapidamente, podendo causar a morte do animal se nenhuma terapia for instituída, mas respondem positivamente a quimioterapia, no entanto, como consequência de seu elevado potencial para mutações podem desenvolver facilmente resistência, que acaba por favorecer a sobrevivência de células com alto índice mitótico (OLIVEIRA, 2014; MESSICK, 2009).

Existem quatro principais localizações anatômicas para o linfoma: multicêntrico, alimentar, mediastínico e extranodal (cutâneo, sistema nervoso central e outros). Em cães as mais comuns são: multicêntrica com $80 \%$ de prevalência, alimentar, mediastínica, e por último cutâneo. Os linfomas que acometem olho, sistema nervoso central, bexiga, coração e cavidade nasal, classificados como extranodais primários, são os de menor incidência (OLIVEIRA, 2014). 
Os sinais clínicos apresentados pelos cães com linfoma multicêntrico são variáveis, por dependerem do órgão onde o tumor está localizado, mas, comumente, incluem sinais inespecíficos como: anorexia, apatia, perda de peso, caquexia, desidratação, febre, palidez de mucosas e icterícia (FIGHERA etal., 2006). Os achados clínicos mais consistentes do linfoma multicêntrico são linfoadenopatia indolor generalizada, com possível envolvimento de medula óssea, hepatomegalia e esplenomegalia (SILVA; SEQUEIRA, 2016). Os linfonodos afetados apresentam-se com mobilidade livre e podem gerar edema em caso de obstrução mecânica da drenagem linfática (NELSON; COUTO, 2006).

Os cães raramente apresentam apenas um linfonodo aumentado, o que causa dificuldade no diagnóstico desta neoplasia, uma vez que a linfoadenopatia localizada é mais comumente associada com inflamação local, hiperplasia linfoide, ou tumores metastáticos. Apesar de poucas enfermidades clínicas causarem a linfoadenopatia generalizada que podem gerar confusão com o diagnóstico clinico, os diagnósticos diferenciais do linfoma multicêntrico incluem: histoplasmose, blastomicose, leishmaniose, erlichiose, mieloma múltiplo, leucemia mieloide aguda e lúpus eritematoso sistêmico (FIGHERA et al., 2006).

Oliveira (2014) e Cápua et al. (2011) referem que nos cães acometidos pelo linfoma multicêntrico podem ser encontradas algumas anormalidades hematológicas, que, contudo, são igualmente variáveis. A alteração mais comum nesses pacientes é a anemia, sendo, em sua grande maioria, normocítica, normocrômica não regenerativa, porém o perfil hematológico também pode ser normal, e quando há o envolvimento da medula óssea pode apresentar trombocitopenia, neutropenia, linfocitose e presença de precursores de linfócitos imaturos e linfócitos atípicos. Quando há suspeita de linfoma o acompanhamento hematológico dos animais passa a ser fundamental tanto para o estabelecimento de um registro inicial para comparações futuras, quanto para auxiliar no estadiamento da doença, pois, de acordo com Cápua et al. (2011), a presença da anemia no momento do diagnóstico do linfoma foi associada com menor tempo de sobrevida para os cães.

O acompanhamento do perfil bioquímico é importante, pois pode refletir na forma anatômica de linfoma ou em síndromes paraneoplásicas. A avaliação deve conter as enzimas hepáticas, ureia e creatinina para avaliação renal, a quantificação de eletrólitos como o cálcio e caso o nível das proteínas totais estejam aumentadas é ideal realizar a eletroforese de proteínas séricas para identificar a gamopatia monoclonal (ALLISON, 2015; OLIVEIRA, 2014).

Os tumores podem produzir sinais em locais distantes dos tumores primários ou de onde estão localizadas as suas metástases, esses sinais são chamados de síndrome paraneoplásica. Essa síndrome pode acontecer devido à produção de substâncias pelo tumor que direta ou indiretamente causam sinais distintos. A depleção de substâncias normais leva a manifestações paraneoplásicas, ou a resposta do hospedeiro frente ao tumor que resulta em uma síndrome. Proteínas derivadas de tumores responsáveis pela síndrome paraneoplásica foram identificadas e incluem fatores de crescimento e citocinas, tais como interleucina-1 e fator de necrose tumoral (DHALIWAL; KITCHELL; MESSICK, 2003).

As síndromes paraneoplásicas comumente relatadas associadas ao linfoma canino são: alterações hematológicas que podem surgir durante a proliferação neoplásica como anemia normocítica normocrômica não regenerativa, anemia hemolítica e trombocitopenia, supostamente devido ao fato das células perderem a capacidade de supressão contra as doenças autoimunes, colaborando dessa forma para a destruição das células e desenvolvendo as citopenias (PROENÇA, 2009); hipercalcemia é a mais registrada e pode ser explicada pela produção de um peptídeo semelhante ao paratormônio das células neoplásicas, assim como em consequência da liberação, por parte dos linfócitos neoplásicos, de fatores como o fator ativador de osteoclastos aumentando a reabsorção óssea (FIGHERA et al., 2002), além de poder estar associada a outros fatores como interleucina 1 , fator de necrose tumoral $\alpha$, fator de crescimento tumoral $\beta$ e análogo de vitamina D (VAIL; YOUNG, 2007), promovendo assim o aumento sérico por retirar cálcio dos ossos liberando-o na circulação (ROCHA; SUZANO; RODRIGUES, 2010). Esta síndrome pode aparecer clinicamente associada a sinais inespecíficos como poliúria e polidipsia, vômitos e anorexia, diarreia e obstipação, até mesmo arritmias cardíacas, podem levar a nefropatias 
como consequência da disfunção tubular renal e urolitíase (FIGHERA et al., 2002; WELLER; HOFFMAN, 1992); Além dos relatos de neuropatia, miastenia gravis, e caquexia cancerosa (DHALIWAL; KITCHELL; MESSICK, 2003).

Casos de cura do linfoma canino são raros $(<10 \%)$, mas apesar disso, frequentemente consegue-se uma resposta positiva a terapêutica mantendo boa qualidade de vida ao animal durante o período de remissão. 0 prognóstico do estadiamento do linfoma baseia-se em critérios clínicos e clinico-patológicos que permitem a determinação da extensão da doença. Esta classificação divide-se em cinco estádios e dois subestádios: Estádio I - o envolvimento limitado a tecido linfoide de um órgão, descartando a medula óssea, ou limita-se a um só linfonodo; Estádio II - envolvimento dos linfonodos de uma determinada região podendo ser acompanhado ou não do acometimento das tonsilas; Estádio III - Envolvimento generalizado dos linfonodos; Estádio IV - Envolvimento de baço e fígado, com ou sem estádio III; Estádio V - Expressões hematológicas e envolvimento da medula óssea e/ou extranodal, com ou sem os estádios de I a IV; Substágio a - sem sinais sistêmicos; Substágio b - com sinais sistêmicos (COUTO, 2015; PROENÇA, 2009).

Sánchez et al. (2019) relataram recentemente que os tutores de cães diagnosticados com linfoma canino levaram em média 50 dias para procurar um atendimento médico veterinário após suspeitar de alguma anormalidade, 64\% (17/27) relataram ter como queixa principal a palpação de um linfonodo aumentado, corroborando com o que foi descrito por Silva e Sequeira (2016), sendo que os outros animais apresentaram sinais inespecíficos como perda de peso, deficiência de visão, prurido e queda de pelos em um animal diagnosticado posteriormente com linfoma cutâneo, ascite, orquite, e um dos cães recebeu o diagnóstico após linfadenectomia em razão de neoplasia mamária. Ao chegar ao atendimento, em 70,3\% (19/27) dos casos os animais foram classificados no estádio clínico IV, e apenas 3,7\% (1/27) no estádio I.

A quimioterapia surge como a modalidade terapêutica mais frequente para o linfoma multicêntrico canino, devido ao fato de tratar-se de uma doença sistêmica. Apesar da baixa taxa de cura, a sobrevida de animais tratados situa-se entre 12 a 16 meses, contudo 25\% dos casos podem sobreviver por dois anos após o diagnóstico (COUTO, 2015). Saliente-se, no entanto, que Sánchez et al. (2019) citaram que os animais não tratados morreram em duas semanas.

A remissão da doença é considerada em $100 \%$ quando há redução de todas as alterações inicialmente detectadas, remissão parcial quando há redução de 50 a 100\% das alterações, já as considerações em doença estável são feitas a partir do momento em que apresenta menos de $50 \%$ de redução ou não são evidenciadas alterações e/ou não foram apresentadas novas lesões neoplásicas. Já a doença é classificada como progressiva quando há novas lesões neoplásicas ou há o aumento das alterações iniciais em 25\%. Apesar disto, Médicos Veterinários oncologistas admitem que é mais importante a abordagem paliativa do que a obtenção de cura (PROENÇA, 2009).

Alguns fatores interferem no prognóstico de cães que apresentam linfoma, tais como: a idade, alguns veterinários oncologistas acreditam que a pouca idade pode influenciar negativamente no prognóstico; a classificação histológica, sendo que os linfomas de baixo grau são mais favoráveis e o linfoma T é apontado como o de pior prognóstico; na imunofenotipagem, as sobrevivências são mais correlacionadas ao imunofenótipo de células B; e a exposição à esteroides, que tem efeitos negativos na sobrevida do animal e no sucesso da remissão do tumor, isso se deve a indução da resistência a outros agentes quimioterápicos que podem ser utilizados nos protocolos de tratamento do linfoma (DHALIWAL; KITCHELL; MESSICK, 2003). 


\section{Considerações finais}

O linfoma multicêntrico canino é uma neoplasia de alta morbidade e mortalidade dentro da clínica, os animais acometidos apresentam um prognóstico reservado e é de extrema importância a conscientização e capacitação dos médicos veterinários para o domínio dos aspectos clinicopatológicos, bem como, das alterações laboratoriais da patologia. Deve-se conhecer as classificações histopatológicas para uma melhor interpretação dos laudos e dessa maneira instituir a terapia precisa e, ainda, aumentar as expectativas de sucesso frente a uma doença comumente encontrada em rotina. A partir de diagnósticos precoces, as taxas de sobrevida dos cães poderão ser ampliadas, favorecendo o prognóstico e a qualidade de vida do animal.

\section{Referências}

ALLISON, R. W. Avaliação laboratorial das proteínas do plasma e do soro sanguíneo. In: THRALL, M. A. et al. Hematologia e bioquímica clínica veterinária. 2. ed. São Paulo: ROCA, p. 404-407, 2015.

CÁPUA, M. L. B. et al. Linfoma canino: clínica, hematologia e tratamento com o protocolo de MadisonWisconsin. Ciência Rural, v. 41, p. 1245-1251, 2011. Disponível em: https://www.scielo.br/pdf/ cr/2011nahead/a4011cr3979.pdf. Acesso em: 2 fev. 2018.

COUTO, C. G. Princípios do tratamento do câncer. In: NELSON, R. W.; COUTO, C. G. Medicina interna de pequenos animais. 5. ed. Rio de Janeiro: Elsevier, p. 1134-1137, 2015.

DHALIWAL, R. S.; KITCHELL, B. E.; MESSICK, J. B. Canine Iymphosarcoma: clinical features. Compendium on continuing education for the practicing veterinarian, v. 25, n. 8. Aug. 2003. Disponível em: https://pdfs.semanticscholar.org/e6a3/007e12ffeebc3095592b654f5ecf24293a01.pdf. Acesso em: 9 maio 2018.

DICKINSON, R. M. Canine lymphosarcoma: overcoming diagnostic obstacles and introduction to the latest diagnostic techniques. The Canadian Veterinary Journal, v. 49, n. 3, p. 305-308, 2008. Disponível em: https://www.ncbi.nlm.nih.gov/pmc/articles/PMC2249731/. Acesso em: 9 maio 2018.

FERREIRA, T. M. V.; AZEVEDO, L. M.; LEITE, A. K. R. M. Aspectos clínico-laboratoriais de linfoma em cão: relato de caso. Revista Brasileira de Higiene e Sanidade Animal, v. 9, n. 4, p. 675-688, 2015. Disponível em: http://www.higieneanimal.ufc.br/seer/index.php/higieneanimal/article/view/282/1003. Acesso em: 2 fev. 2018.

FIGHERA, R. A.; SOUZA, T. M.; BARROS, C. S. L. Linfossarcoma em cães. Ciência Rural, v. 32, n. 5, p. $895-$ 899, 2002. Disponível em: https://www.scielo.br/pdf/cr/v32n5/11884.pdf. Acesso em: 5 maio 2018.

FIGHERA, R. A. et al. Aspectos clinicopatológicos de 43 casos de linfoma em cães. MEDVEP: Revista Científica de Medicina Veterinária - Pequenos Animais e Animais de Estimação, v. 4, n. 12, p. 139-146, 2006. Disponível em: http://www.rafaelfighera.com.br/wp-content/uploads/2014/06/rafaelfighera_ linfoma-em-caes.pdf. Acesso em: 3 fev. 2018.

GOOD, D. J.; GASCOYNE, R. D. Classification of Non-Hodgkin's Lymphoma. Hematology Oncology Clinics of North America, v. 22, p. 781-805, 2008. . Disponível em: https://doi.org/10.1016/j.hoc.2008.07.008. Acesso em 24 ago. 2020.

GREENLEE, P. G. et al. Lymphomas in dogs: a morphologic, immunologic, and clinical study. Cancer. Philadelphia, v. 66, p. 480-490, 1990. Doi: 10.1002/1097-0142(19900801)66:3<480::aidcncr2820660314>3.0.c0;2-x. Disponível em: https://pubmed.ncbi.nlm.nih. gov/2364361/\#: : text=Several\%20different\%20morphologic\%20types\%20 of,cell\%20 (centroblastic)\%20and\%20immunoblastic. Acesso em: 3 fev. 2018. 
HARRIS, N. L. et al. Revised European - American Classification of lymphoid neoplasms: a proposal from the International Lymphoma Study Group. Blood, v. 84, n. 5, p. 1361-1392, 1994.

JAFFE, E. S.et al. World Health Organization Classification of Tumors: pathology and genetics. Tumours of haematopoietic and lymphoid tissues. IARCPress: Lyon, 351 p, 2001.

KIMURA, K. C. Linfoma canino: papel do meio ambiente. 2012. 139 f. Tese (Doutorado em Ciências) Faculdade de Medicina Veterinária e Zootecnia, Universidade de São Paulo, São Paulo, 2012. Disponível em: https://www.teses.usp.br/teses/disponiveis/10/10133/tde-05082013-165249/publico/KATIA CRISTINA_KIMURA_Original.pdf. Acesso em: $10 \mathrm{fev} .2018$.

LENNERT, K.; FELLER, A. C. Histopathology of Non-Hodgkin's lymphomas. 2. ed. Berlim: SpringerVerlag, 312 p, 1992.

MESSICK, J. B. Os Linfonodos. In: COWELL, R. L.; TYLER, R. D.; MEINKOTH, J. H.; DENICOLA, D. B. Diagnóstico citológico e hematologia de cães e gatos. São Paulo: MedVet, p. 185-188, 2009.

NELSON, R. W.; COUTO, C. G. Medicina interna de pequenos animais. Rio de Janeiro: Elsevier, p. 10871096, 2006.

OLIVEIRA, A. I. A. Linfoma canino e felino: revisão bibliográfica e estudo de 3 casos clínicos. 72 f. 2014. Dissertação (Mestrado Integrado em Medicina Veterinária) - Faculdade de Medicina Veterinária, Universidade de Lisboa, Lisboa, p. 27-31.2014. Disponível em: https://www.repository.utl.pt/ bitstream/10400.5/7819/1/Linfoma\%20Canino\%20e\%20Felino\%20-\%20Revis\%C3\%A30\%20 bibliogr\%C3\%A1fica\%20e\%20estudo\%20de\%203\%20casos\%20cl\%C3\%ADnicos.pdf. Acesso em: 6 fev. 2018.

PROENÇA, A. R. D. S. G. Linfoma maligno multicêntrico canino. 115 f. Dissertação (Mestrado em Medicina Veterinária) - Faculdade de Medicina Veterinária, Universidade Técnica de Lisboa, Lisboa, 2009. Disponível em: https://www.repository.utl.pt/bitstream/10400.5/1406/1/Linfoma\%20maligno\%20 multic\%C3\%AAntrico\%20canino.pdf. Acesso em: 4 fev. 2018.

ROCHA, A. A.; SUZANO, S. M. C.; RODRIGUES, R. L. Classificação histológica e imunoistoquímica em três casos de linfoma canino. Revista Eletrônica Novo Enfoque, v. 9, n. 9, p. 32-47, 2010. Disponível em: https://docplayer.com.br/692764-Classificacao-histologica-e-imunoistoquimica-em-tres-casos-delinfoma-canino.html. Acesso em: 3 fev. 2018.

SÁNCHEZ, D. et al. Canine lymphoma: pathological and clinical characteristics of patients treated at a referral hospital. Veterinaria México AO, v. 6, n. 2, Apr.-June, 2019. Disponível em: http:// veterinariamexico.unam.mx/index.php/vet/article/view/495/615. Acesso em: 26 nov. 2019.

SILVA, M. C. L.; SEQUEIRA, J. L. Linfoma canino: revisão de literatura com ênfase no linfoma difuso de grandes células B. Veterinária e Zootecnia, v. 23, n. 4, p. 561-576, 2016. Disponível em: https://rvz. emnuvens.com.br/rvz/article/download/407/222/. Acesso em: 12 fev. 2018.

TESKE, E. Canine malignant lymphoma: a review and comparion whit human non-Hodgkin's lymphoma. Veterinary Quarterly, Dordrecht, v. 16, n. 4, p. 209-219, 1994.

VAIL, D. M.; MACEWEN, E. G.; YOUNG, K. M. Canine lymphoma and lymphoid leukemias. In: WITHROW, S. J.; MACEWEN, E. G. Small animal clinical oncology. 3rd ed. Philadelphia: Saunders Company, p. 55890,2001 .

VAIL, D. M.; OGILVIE, G. K. Neoplasias Linfóides. In: BIRCHARD, S. J.; SHERDING, R. G. Manual Saunders clínica de pequenos animais. 2. ed. São Paulo: Roca, p. 227-236, 2003.

VAIL, D. M.; YOUNG, K. M. Canine lymphoma and Iymphoid leukemia. In: WITHROW, S. J.; VAIL, D. M. Withrow \& MacEwen's small animal clinical oncology. Philadelphia: W. B. Saunders Company, cap. 31, p. 699-733, 2007. 
VALERIANO, K. C. et al. Enucleação decorrente de linfoma ocular em cão: relato de caso. Eventos Científicos da Fundação Educacional de Ituverava, v. 1, n. 1, p. 148, 2017.

VALLI, V. E. et al. Histological classification of hematopoietic tumors of domestic animals. In: World Health Organization International Histological Classification of Tumors in Domestic Animals. 2.ed. Washington, DC: Armed Forced Institute of Pathology, 190 p, 2002.

VALLI, V. E. The evolution of classification systems for hematopoietic neoplasms. In: VALLI, V. E. (Ed.). Veterinary comparative hematopathology. Ames: Blackwell Publishing, p. 3-7, 2007.

VIEIRA, M. C. Perfil de proteínas séricas em cães com linfoma multicêntrico de imunofenótipo B ou T: correlação com fatores prognósticos. 2013. 92 f. Tese (Doutorado) - Faculdade de Ciências Agrárias e Veterinárias, Universidade Estadual Paulista (Unesp), Jaboticabal. 2013. Disponível em: https:// repositorio.unesp.br/bitstream/handle/11449/104618/000722350.pdf? sequence=1\&isAllowed=y. Acesso em: 5 fev. 2018.

WELLER, R. E.;HOFFMAN, W. E. Renal function in dogs with lymphosarcoma and associated hypercalcemia. Journal of Small Animal Practice, London, v. 33, n. 1, p. 61-66, 1992. 